\title{
Metformin in gestational diabetes mellitus: predictors of poor response
}

\author{
Inês Gante ${ }^{1, *}$, Luís Melo², Jorge Dores ${ }^{3,4}$, Luísa Ruas ${ }^{4,5}$ and Maria do Céu Almeida ${ }^{1,4}$ \\ ${ }^{1}$ Department of Obstetrics, Coimbra Hospital and Universitary Centre, Coimbra, Portugal, ${ }^{2}$ Faculty of Medicine, \\ University of Coimbra, Coimbra, Portugal, ${ }^{3}$ Department of Endocrinology, Porto Hospital Centre, Porto, Portugal, \\ ${ }^{4}$ Diabetes and Pregnancy Study Group of the Portuguese Society of Diabetology, Lisbon, Portugal, ${ }^{5}$ Department of \\ Endocrinology, Coimbra Hospital and Universitary Centre, Coimbra, Portugal \\ *(I Gante and L Melo contributed equally to this work)
}

Correspondence should be addressed to I Gante

Email

inesrcgante@gmail.com

\begin{abstract}
Objective: Metformin can be regarded as a first-line treatment in gestational diabetes mellitus (GDM) due to its safety and effectiveness. However, a proportion of women do not achieve adequate glycemic control with metformin alone. We aim to identify predictors of this poor response to metformin.

Design and methods: Retrospective multicentre cohort study of women with GDM who started metformin as first-line treatment. The assessed cohort was divided into a metformin group and metformin plus insulin group. Biometric and demographic characteristics, glycemic control data, obstetric, neonatal and postpartum outcomes were compared between groups and analysed in order to identify predictors of poor response to metformin. Data were analysed using STATA, version 13.1 .

Results: Of the 388 women enrolled in the study, 135 (34.8\%) required additional insulin therapy to achieve the glycemic targets. Higher age (aOR: $1.08(1.03-1.13), P=0.003)$, higher pre-pregnancy body mass index (BMI) (1.06 $(1.02-1.10), P=0.003)$ and earlier introduction of metformin $(0.89(0.85-0.94), P<0.001)$ were independent predictors for insulin supplementation. Regarding all the analysed outcomes, only cesarean delivery rates and postpartum glucose levels were higher in women requiring insulin supplementation.

Conclusions: Although almost $35 \%$ of women did not achieve adequate glycemic control with metformin, insulin supplementation was not associated with poor neonatal outcomes. Higher age, higher pre-pregnancy BMI and earlier introduction of metformin could be used as predictors of poor response to metformin.
\end{abstract}

\section{Introduction}

The incidence of gestational diabetes mellitus (GDM) has risen worldwide over the past few decades, mainly due to an increase in maternal age and in obesity-induced insulin resistance $(1,2,3)$.

It is estimated that GDM affects over $10 \%$ of pregnancies, depending on the diagnostic methods used and the population studied $(1,3,4,5)$. In Portugal, in 2014, almost $6.7 \%$ of pregnancies were complicated by GDM (6).

GDM is an independent risk factor for obstetric complications, such as preterm labour, preeclampsia, large for gestational age (LGA) and macrosomic newborns,

www.eje-online.org

https://doi.org/10.1530/EJE-17-0486
(C) 2018 European Society of Endocrinology Printed in Great Britain growth restriction, birth trauma, cesarean section and neonatal hypoglycaemia (7).

It is known that adequate glycemic control minimizes the risk of obstetric complications. When lifestyle modifications (dietary and physical activity) are not sufficient to achieve appropriate glucose levels, pharmacological therapy is required $(4,8)$.

Insulin is historically the first-line treatment because of its safety and effectiveness.

Oral hypoglycaemic agents, especially metformin, are reliable in the management of blood glucose and 
are a safe and cheaper alternative $(8,9,10,11,12,13$, 14). Furthermore, metformin is associated with lower gestational weight gain (GWG), had a reduced risk of hypoglycaemia and showed no compromise of the motor, linguistic and social development in recent studies (15).

Despite all these benefits, in up to $46 \%$ of women, adequate glycemic control is not achieved with metformin alone, requiring insulin supplementation (14).

The aim of this study was to identify predictors of poor response to metformin alone. Additionally, we want to evaluate glycemic control data and obstetric, neonatal and postpartum outcomes among women with GDM, treated with metformin alone versus metformin plus insulin.

\section{Subjects and methods}

\section{Study design}

A retrospective multicentre cohort study in Portuguese public hospitals between January 2014 and December 2015 of pregnant women with GDM treated with metformin as first-line pharmacologic therapy. Data were obtained from the analysis of the National Registry of GDM concerning these years $(n=5352)$ after consent from the Diabetes and Pregnancy Study Group of the Portuguese Society of Diabetology (Sociedade Portuguesa de Diabetologia), which is responsible for this database. Data were collected by the multidisciplinary teams of Obstetrics and Diabetology in 25 Portuguese public sector health institutions (among the total of 44 invited to collaborate), comprising all Portuguese continental districts. Considering the number of Portuguese women with GDM in 2014-2015 ( $n=9174)$, the total registry participation rate was $58.3 \%$.

Subsequently, all data sets were blinded relatively to the patients and hospital identification, ensuring anonymity of the collected data. This study complies with the Declaration of Helsinki on medical protocol and ethics. Each participating hospital's institutional review board approved data collection. Given the retrospective nature of this study and its anonymity, written consent was not required.

\section{Study subjects}

The National Registry of GDM includes all women that fulfil GDM criteria and whose follow-up was performed in Portuguese public health care institutions. However, only women without satisfactory glucose levels despite adequate non-pharmacological therapy and who started the hypoglycaemic therapy with metformin (as first line) were included in this study.

The GDM diagnosis was established according to the Portuguese Directorate-General of Health (Direção Geral de Saúde) criteria that were based on the International Association of Diabetes and Pregnancy Study Groups' criteria: universal fasting plasma glucose in the 1st trimester and $75 \mathrm{~g}$ two-hour oral glucose tolerance test (OGTT) between 24 and 28 gestational weeks (16).

After diagnosis, during the first specialized appointment, baseline data were collected and all patients were counselled about diet and exercise in order to achieve their glycemic targets.

The goal of therapy was to reduce fasting glucose to $<95 \mathrm{mg} / \mathrm{dL}$ and the 1 -h postprandial level to $<120 \mathrm{mg} / \mathrm{dL}$ in more than $80 \%$ of the glucose measurements.

Metformin as first-line pharmacological therapy was started if the glycemic targets were not achieved in 1-2 weeks of non-pharmacological therapy. It was prescribed in an initial daily dose of $500 \mathrm{mg}$ up to a maximum daily dose of $2500 \mathrm{mg}$. Insulin supplementation was initiated if the glycemic targets were not achieved in 2 weeks despite the increase in metformin dose. All women stopped the antidiabetic medication at delivery.

The patients included in this study were divided into two groups: those who respond to metformin alone and those who needed insulin supplementation.

\section{Study outcomes}

The data analysed included baseline biometric and demographic characteristics; glycemic control data (such as hemoglobin A1c (HbA1c), maximum daily dose of metformin and, for those that needed escalation of therapy, the timing of that decision); obstetric and neonatal outcomes and postpartum OGTT. For the characterization of the neonates weight, we used Cole Curves and considered 90th percentile and 10th percentile for gestational age as upper and lower limits, respectively, for appropriate for gestational age (AGA) newborns (17).

The postpartum OGTT was established 6-8 weeks after delivery with a two-hour 75 g OGTT according to the WHO criteria (18).

\section{Statistical analysis}

Categorical data were analysed by the chi-squared test or Fisher exact probability test whenever cell frequencies were $<5$. Continuous data were compared using Student's $t$-test. 
Based on the data from the univariate analysis, multivariate analysis was performed to identify independent parameters for metformin patients requiring insulin supplementation. This analysis was performed and adjusted odds ratios (aOR) were calculated using stepwise backward logistic regression models, adjusted for the following covariates: age, pre-pregnancy body mass index (BMI), first or second trimester GDM and gestational age at introduction of metformin.

Data are expressed as proportions, means $( \pm$ S.D. of the mean), aOR and 95\% confidence intervals (CI).

All calculations were performed with STATA version 13.1 software. The probability of error ( $P$ value) was considered significant when $<0.05$. Statistical significance was accepted when the 95\% CI did not contain one (regression analyses).

\section{Results}

Between January 2014 and December 2015, 388 pregnant women with GDM initiated pharmacological therapy with metformin.

Globally, 73.7\% ( $n=286)$ were Portuguese, the mean age was $33.7 \pm 5.4$ years old, the mean pre-pregnancy BMI was $30.0 \pm 6.5 \mathrm{~kg} / \mathrm{m}^{2}, 44.6 \%(n=173)$ had the diagnosis in the 1st trimester and the start of metformin was at $27.3 \pm 6.6$ gestational weeks. From this cohort, 34.8\% $(n=135)$ needed insulin supplementation.

\section{Baseline characteristics}

As shown in Table 1, both a higher maternal age (35.2 vs 32.9 years old, $P<0.001$ ) as a higher pre-pregnancy BMI (31.8 vs $29.0 \mathrm{~kg} / \mathrm{m}^{2}, P<0.001$ ) were strongly associated with the need for insulin supplementation. Thereby, obese women needed insulin supplementation more frequently (42.1\% ( $n=83)$ vs $27.2 \%(n=52), P=0.002)$.

The OGTT showed higher values in metformin + insulin group; however, only the fasting glucose levels had statistically significant differences $(85.4$ vs $92.7 \mathrm{mg} / \mathrm{dL}$, $P<0.001$ ) (Table 1).

Earlier GDM diagnosis (17.5 vs 20.3 weeks, $P=0.003)$ and earlier metformin introduction (24.3 vs 28.8 weeks, $P<0.001$ ) were associated with failure of metformin monotherapy, leading to the need for insulin supplementation.

Despite only 5 pregnancies being multiple, 4 of them $(80 \%)$ needed insulin supplementation $(P=0.05)$.

\section{Glycemic control data}

Particularly in the 3rd trimester, higher HbA1c values were associated with the need for additional therapy with insulin (5.5 vs $5.2 \%, P<0.001)$. Also, higher maximum daily dose of metformin was associated with escalation of therapy (1858.8 vs $1384.6 \mathrm{mg} /$ day, $P<0.001$ ) (Table 2).

Table 1 Baseline characteristics and demographics of women with GDM treated with metformin alone versus metformin and insulin. Proportions were compared by the chi-squared test or Fisher exact probability test and means were analysed with Student's $t$-test.

Portuguese, $n(\%)$
Brazilian, $n(\%)$
Other nationality, $n(\%)$
Age (years)
Family history of diabetes, $n .(\%)$
GDM in previous pregnancy, $n(\%)$
Pre-pregnancy BMI (kg/m²)
1st trimester fasting glucose (mg/dL)
Diagnosis in the 1st trimester, $n(\%)$
OGTT (mg/dL)
Fasting
$\quad 1$ h
2 h
Diagnosis (GW)
Weight gain until diagnosis (kg)
1st specialized appointment (GW)
HbA1c (1st specialized appointment) (\%)
Introduction of metformin (GW)

\begin{tabular}{c}
\hline Metformin group $(n=253)$ \\
\hline $184(72.7 \%)$ \\
$8(3.2 \%)$ \\
$61(24.1 \%)$ \\
$32.9 \pm 5.4$ \\
$114(46.5 \%)$ \\
$25(16.9 \%)$ \\
$29.0 \pm 6.3$ \\
$95.6 \pm 10.8$ \\
$101(39.9 \%)$ \\
$85.4 \pm 11.41$ \\
$171.4 \pm 32.2$ \\
$144.6 \pm 33.8$ \\
$20.3 \pm 8.1$ \\
$5.2 \pm 5.3$ \\
$24.1 \pm 7.6$ \\
$5.3 \pm 0.4$ \\
$28.8 \pm 6.1$
\end{tabular}

\begin{tabular}{c}
\hline Metformin + insulin group $(n=135)$ \\
$102(75.6 \%)$ \\
$10(7.4 \%)$ \\
$23(17.0 \%)$ \\
$35.2 \pm 5.0$ \\
$53(42.7 \%)$ \\
$21(26.6 \%)$ \\
$31.8 \pm 6.6$ \\
$97.6 \pm 10.9$ \\
$72(53.3 \%)$ \\
$92.7 \pm 15.3$ \\
$176.8 \pm 33.1$ \\
$152.9 \pm 40.0$ \\
$17.5 \pm 8.0$ \\
$3.9 \pm 6.1$ \\
$22.4 \pm 7.7$ \\
$5.4 \pm 0.5$ \\
$24.3 \pm 6.6$
\end{tabular}

\begin{tabular}{l}
\hline $\boldsymbol{P}$ value \\
\hline 0.547 \\
0.058 \\
0.107 \\
$<0.001$ \\
0.490 \\
0.084 \\
$<0.001$ \\
0.203 \\
0.011 \\
\\
$<0.001$ \\
0.297 \\
0.141 \\
0.003 \\
0.102 \\
0.039 \\
0.158 \\
$<0.001$
\end{tabular}

GW, gestational weeks. 
Table 2 Glycemic control data and obstetric outcomes of women with GDM treated with metformin alone versus metformin and insulin. Proportions were compared by the chi-squared or Fisher exact test and means were analysed with Student's $t$-test.

\begin{tabular}{l}
\hline 3rd trimester HbA1c (\%) \\
Maximum daily dose of metformin (mg) \\
Time between metformin and insulin therapy (GW) \\
Gestational weight gain after diagnosis (kg) \\
Gestational hypertension, $n(\%)$ \\
Preeclampsia, $n(\%)$ \\
Hydramnios, $n(\%)$ \\
Induced labour, $n(\%)$ \\
Eutocic delivery, $n(\%)$ \\
Instrumental vaginal delivery, $n(\%)$ \\
Cesarean delivery, $n(\%)$ \\
Urgent cesarean, $n(\%)$ \\
Elective cesarean, $n(\%)$ \\
Delivery (GW) \\
Premature birth, $n(\%)$
\end{tabular}

\begin{tabular}{c} 
Metformin group $(n=253)$ \\
\hline $5.2 \pm 0.5$ \\
$1384.6 \pm 723.6$ \\
- \\
$3.0 \pm 4.5$ \\
$21(8.7 \%)$ \\
$7(3.0 \%)$ \\
$12(5.0 \%)$ \\
$104(55.9 \%)$ \\
$130(53.5 \%)$ \\
$36(14.8 \%)$ \\
$77(31.7 \%)$ \\
$37(14.6 \%)$ \\
$40(15.8 \%)$ \\
$38.3 \pm 1.3$ \\
$18(7.1 \%)$
\end{tabular}

\begin{tabular}{ccc}
\hline Metformin + insulin group $(n=135)$ & & $P$ value \\
\cline { 1 - 1 } $5.5 \pm 0.5$ & & $<0.001$ \\
$1858.8 \pm 1010.1$ & & $<0.001$ \\
$4.0 \pm 4.2$ & - & 0.130 \\
$2.1 \pm 4.9$ & & 0.510 \\
$14(10.8 \%)$ & 0.137 \\
$8(6.2 \%)$ & 0.880 \\
$7(5.4 \%)$ & 0.065 \\
$59(67.8 \%)$ & 0.010 \\
$51(39.5 \%)$ & 0.667 \\
$17(13.2 \%)$ & 0.003 \\
$61(47.3 \%)$ & 0.531 \\
$23(17.0 \%)$ & 0.006 \\
$37(27.4 \%)$ & 0.870 \\
$38.4 \pm 1.1$ & 0.656 \\
$8(5.9 \%)$ &
\end{tabular}

GW, gestational weeks.

Women requiring additional therapy with insulin to achieve desirable glucose levels started taking the insulin supplementation $4.0 \pm 4.2$ weeks after metformin initiation, at $28.3 \pm 5.9$ weeks of pregnancy. The mean insulin dose needed was $22.2 \pm 20.5 \mathrm{IU}$.

\section{Independent predictors for insulin supplementation}

As shown in Table 3, a higher age (aOR: 1.08 (1.03-1.13)), a higher pre-pregnancy BMI (aOR: 1.06 (1.02-1.10)) and an earlier introduction of metformin (aOR: 0.89 (0.850.94)) were independently associated with the need for insulin supplementation.

Specifically, maternal age $\geq 35$ years (aOR: 2.30 (1.473.59), $P<0.001$ ), pre-pregnancy obesity (aOR: 1.90 (1.212.98), $P=0.005$ ) and introduction of metformin before 24 weeks of gestation (aOR: 3.27 (1.77-6.05), $P<0.001$ ) were independently associated with an increased risk of insulin supplementation.

\section{Obstetric and neonatal outcomes}

Obstetric and neonatal outcomes are summarized in Tables 2 and 4 .

One abortion was reported in the metformin group in a woman with antiphospholipid syndrome, and none occurred in the metformin +insulin group $(P=0.644)$. There were no cases of foetal death in our cohort.

Although without statistically significant differences, we noted a slight tendency towards less obstetric complications in the metformin group (Table 2). Additionally, cesareans were more common in metformin+insulin group $(47.3 \%$ vs $31.7 \%$ in the metformin group, $P=0.003)$, mainly due to the higher percentage of elective cesarean $(27.4 \%$ vs $15.8 \%, P=0.006)$ (Table 2).

The two groups were comparable with respect to weight at birth, Apgar scores and neonatal morbidities (Table 4). Using the Cole Curves, the frequency rate of AGA was $75.8 \%(n=94)$ in the metformin+insulin group vs $84.3 \%(n=198)$ in the metformin group $(P=0.051)$ : $8.1 \%(n=10)$ vs $4.7 \%(n=11)(P=0.194)$ were small for gestational age (SGA) and $16.1 \%(n=20)$ vs $11.1 \%(n=26)$ $(P=0.172)$ were large for gestational age (LGA).

\section{Postpartum outcomes}

Women in the metformin+insulin group were more likely to have higher postpartum glucose levels ((fasting: $95.7 \mathrm{mg} / \mathrm{dL}$ vs $88.0 \mathrm{mg} / \mathrm{dL}, \quad P<0.001$ ); (OGTT $-2 \mathrm{~h}$ : $104.1 \mathrm{mg} / \mathrm{dL}$ vs $94.3 \mathrm{mg} / \mathrm{dL}, \quad P=0.019))$. Nevertheless, the postpartum OGTT results did not show differences between the two groups (Table 5).

Table 3 Independent predictors for insulin supplementation.

\begin{tabular}{|c|c|c|}
\hline & aOR $(95 \% \mathrm{Cl})$ & $P$ value \\
\hline Age (years) & $1.08(1.03-1.13)$ & 0.003 \\
\hline Pre-pregnancy BMI $\left(\mathrm{kg} / \mathrm{m}^{2}\right)$ & $1.06(1.02-1.10)$ & 0.003 \\
\hline Introduction of metformin (GW) & $0.89(0.85-0.94)$ & $<0.001$ \\
\hline
\end{tabular}

Multivariate analysis was performed and aORs were calculated using logistic regression models, adjusted for the following covariates: age, pre-pregnancy BMI, first or second trimester GDM and gestational weeks (GW) at the introduction of metformin. 
Table 4 Neonatal outcomes of women with GDM treated with metformin alone versus metformin and insulin. Proportions were compared by the chi-squared or Fisher exact test and means were analysed with Student's $t$-test.

\begin{tabular}{lcc}
\hline & Metformin group $(n=253)$ \\
\hline Neonatal weight at birth $(\mathrm{g})$ & $3197.9 \pm 457.1$ \\
Low birth weight $(<2500 \mathrm{~g}), n(\%)$ & $15(5.9 \%)$ \\
Macrosomia $(\geq 4000 \mathrm{~g}), n(\%)$ & $18(7.1 \%)$ \\
Apgar score at 5 min & $9.8 \pm 0.8$ \\
Total neonatal morbidity, $n(\%)$ & $63(26.5 \%)$ \\
Hypoglycaemia, $n(\%)$ & $18(7.8 \%)$ \\
Hyperbilirubinemia, $n(\%)$ & $41(17.7 \%)$ \\
Respiratory distress, $n(\%)$ & $10(4.3 \%)$ \\
Neonatal intensive care unit admission, $n(\%)$ & $19(8.2 \%)$ \\
\hline
\end{tabular}

\begin{tabular}{|c|c|}
\hline Metformin + insulin group $(n=135)$ & $P$ value \\
\hline $3184.3 \pm 555.8$ & 0.802 \\
\hline $15(11.1 \%)$ & 0.069 \\
\hline $14(10.4 \%)$ & 0.267 \\
\hline $9.9 \pm 0.4$ & 0.139 \\
\hline $26(20.7 \%)$ & 0.218 \\
\hline $9(7.8 \%)$ & 0.991 \\
\hline $20(16.8 \%)$ & 0.839 \\
\hline $4(3.5 \%)$ & 0.711 \\
\hline $6(5.1 \%)$ & 0.295 \\
\hline
\end{tabular}

\section{Discussion}

As shown in other studies, metformin alone may be insufficient to achieve adequate glycemic control in GDM. In our study, $34.8 \%$ required insulin supplementation. This proportion is similar to that reported by Moore and coworkers $(34.7 \%)$ but lower than that showed by Rowan and coworkers (48.3\%) and higher than that by Tertti and coworkers $(18 \%)(14,19,20)$. This reflects the heterogeneity of the populations studied.

Higher age, higher pre-pregnancy BMI and earlier introduction of metformin are the main conditions individualized in our analysis as independent predictors for insulin supplementation.

Similar to our findings, Tertti and coworkers reported that higher age was related to the need for insulin supplementation (20). Specifically, in our study, age above 35 years was associated with more than double the risk of insulin supplementation.

The majority of studies connected obesity and higher BMI with weaker sensitivity to metformin. In particular, lower BMI populations were associated with a better response to metformin alone, as demonstrated by Niromanesh and coworkers, in which the mean BMI was $28.1 \mathrm{~kg} / \mathrm{m}^{2}$ and only $14 \%$ required additional insulin
(21). By contrast, greater BMI populations were associated with a higher proportion of therapy escalation as reported in the MiG trial (14). Our data are in line with previous studies since $42.1 \%$ of obese women required additional insulin and obesity was associated with a doubled risk of insulin supplementation.

Early diagnosis and early introduction of metformin predicted insulin supplementation. Similar to results from McGrath and coworkers and Spaulonci and coworkers, more than a half of the women in our study that required additional therapy with insulin were diagnosed with GDM in the first trimester of pregnancy $(22,23)$. Also, the introduction of metformin before 24 weeks of gestation tripled the risk of insulin supplementation. When additional therapy with insulin was required, the gap between introduction of metformin and insulin supplementation was very variable $(4 \pm 4.2$ weeks), which is similar to the gap reported by McGrath and coworkers (30 \pm 22 days) (22). Third trimester HbA1c and maximum daily dose of metformin were both significantly higher in the group that required additional therapy with insulin. These correlations could be due to the increased insulin resistance during late gestation, since placental growing induces progressive insulin resistance which, associated

Table 5 Postpartum OGTT of women with GDM treated with metformin alone versus metformin and insulin. Proportions were compared by the chi-squared or Fisher exact test and means were analysed with Student's $t$-test.

\begin{tabular}{|c|c|c|c|}
\hline & Metformin group $(n=167)$ & Metformin + insulin group $(n=87)$ & $P$ value \\
\hline \multicolumn{4}{|l|}{ Postpartum OGTT (mg/dL) } \\
\hline Fasting & $88.0 \pm 9.1$ & $95.7 \pm 15.7$ & $<0.001$ \\
\hline $2 \mathrm{~h}$ & $94.3 \pm 28.2$ & $104.1 \pm 32.0$ & 0.019 \\
\hline \multicolumn{4}{|l|}{ Postpartum OGTT result, $n(\%)$} \\
\hline Normal & $151(90.4 \%)$ & $75(86.2 \%)$ & 0.309 \\
\hline Impaired fasting glucose & $3(1.8 \%)$ & $3(3.5 \%)$ & 0.415 \\
\hline Impaired glucose tolerance & $11(6.6 \%)$ & $7(8.1 \%)$ & 0.668 \\
\hline Type 2 diabetes mellitus & $2(1.2 \%)$ & $6(2.4 \%)$ & 0.608 \\
\hline
\end{tabular}


to impaired beta cell function, led to increasingly glucose levels.

Hughes and coworkers found that an increased early pregnancy HbA1c measurement $(\geq 5.9 \%)$ was associated with an increased risk of adverse pregnancy outcomes, including major congenital anomaly, preeclampsia, shoulder dystocia and perinatal death (24). The impact of early detection and active intervention on hyperglycaemia in pregnancy could be responsible for smothering differences on both maternal and foetal outcomes between GDM diagnosed early and later, as we have shown in our study.

Several studies suggest a correlation between higher fasting blood glucose at diagnosis and the need for insulin supplementation $(10,14,22)$. In our study, we only found this correlation with the values of fasting blood glucose in 2nd trimester OGTT.

Regarding the obstetric and neonatal outcomes analysed, the cesarean rate was statistically different between the two groups, due to the higher rate of elective cesareans in the metformin+insulin group (27.4\%). The majority of studies had similar rates of cesarean deliveries between metformin and insulin groups $(14,25,26)$. This suggests that insulin per se might not be a risk factor for cesarean delivery; moreover, in our study, the need for additional therapy with insulin was associated with other known risk factors for cesarean delivery, such as higher BMI and poor glycemic control.

Despite the slightly higher rates of AGA newborns in the metformin group, there were no statistically significant differences in SGA and LGA between the two groups. This corroborates the finding that even in women with inadequate glycemic control with metformin, the gap until escalation of therapy might not determine worse neonatal outcomes.

Although a recent study indicated that short-term metformin therapy does not affect postpartum OGTT results, compared with insulin or diet-only treatments, in our study, the need for additional therapy with insulin was associated with higher glucose levels in the postpartum OGTT (27).

A limitation of this study is the fact that women were followed in 25 different Portuguese hospitals and clinical judgement facing the same situations may have been different despite the well-established standards. Furthermore, the diagnostic criteria for GDM vary between countries and across the time which may induce variations in need for insulin supplementation.

The major strengths of this study are the large sample size and the identification of risk factors for poor response to metformin. Finally, the conclusions of this study are important for clinical practice as they may disclose some questions about metformin use in GDM.

\section{Conclusion}

Metformin can be regarded as an alternative treatment in GDM. Despite being a safe and effective therapy, almost $35 \%$ of the women treated with metformin did not achieve adequate glycemic control with monotherapy.

Higher maternal age, higher pre-pregnancy BMI and earlier need for metformin introduction (due to poor glycemic control with non-pharmacological therapy) were the major independent predictors of insulin supplementation. Also, poor glycemic control indicators (such as higher HbA1c or need for higher daily dose of metformin) were associated with failure of the monotherapy with metformin.

Furthermore, in women requiring insulin supplementation there were higher cesarean rates and higher postpartum glucose levels.

Declaration of interest

The authors declare that there is no conflict of interest that could be perceived as prejudicing the impartiality of this study.

\section{Funding}

This research did not receive any specific grant from any funding agency in the public, commercial or not-for-profit sector.

\section{References}

1 Kennelly MA \& McAuliffe FM. Prediction and prevention of Gestational Diabetes: an update of recent literature. European Journal of Obstetrics and Gynecology and Reproductive Biology 2016202 92-98. (https://doi.org/10.1016/j.ejogrb.2016.03.032)

2 Balani J, Hyer SL, Rodin DA \& Shehata H. Pregnancy outcomes in women with gestational diabetes treated with metformin or insulin: a case-control study. Diabetic Medicine. 200926 798-802. (https://doi. org/10.1111/j.1464-5491.2009.02780.x)

3 American Diabetes Association. Diagnosis and classification of diabetes mellitus. Diabetes Care 201033 (Supplement 1) S62-S69.

4 Cheung NW. The management of gestational diabetes. Vascular Health and Risk Management 20098 153-164. (https://doi. org/10.2147/VHRM.S3405)

5 Ashwal E \& Hod M. Gestational diabetes mellitus: where are we now? Clinica Chimica Acta 2015451 14-20. (https://doi.org/10.1016/j. cca.2015.01.021)

6 Observatório Nacional da Diabetes. Diabetes: Factos e Números - O Ano de 2014 - Relatório Anual do Observatório Nacional da Diabetes, 2015. (available from: https://www.dgs.pt/estatisticas-desaude/estatisticas-de-saude/publicacoes/diabetes-factos-e-numeros-7edicao.aspx)

7 Lowe LP, Metzger BE, Dyer AR, Lowe J, McCance DR, Lappin TRJ, Trimble ER, Coustan DR, Hadden DR, Hod M, et al. Hyperglycemia 
and adverse pregnancy outcome (HAPO) study: associations of maternal A1C and glucose with pregnancy outcomes. Diabetes Care 201235 574-580. (https://doi.org/10.2337/dc11-1687)

8 National Institute for Health and Care Excellence (NICE). Diabetes in pregnancy: management from preconception to the postnatal period - NICE guideline, 2015. (Available from: nice.org.uk/guidance/ng3)

9 Ainuddin JA, Karim N, Zaheer S, Ali SS \& Hasan AA. Metformin treatment in type 2 diabetes in pregnancy: an active controlled, parallel-group, randomized, open label study in patients with type 2 diabetes in pregnancy. Journal of Diabetes Research 20152015 325851. (https://doi.org/10.1155/2015/325851)

10 Ashoush S, El-Said M, Fathi H \& Abdelnaby M. Identification of metformin poor responders, requiring supplemental insulin, during randomization of metformin versus insulin for the control of gestational diabetes mellitus. Journal of Obstetrics and Gynaecology Research 201642 640-647. (https://doi.org/10.1111/jog.12950)

11 Singh KP, Rahimpanah F \& Barclay M. Metformin for the management of gestational diabetes mellitus. Australian and New Zealand Journal of Obstetrics and Gynaecology 201555 303-308. (https://doi.org/10.1111/ajo.12311)

12 Buschur E, Brown F \& Wyckoff J. Using oral agents to manage gestational diabetes: what have we learned? Current Diabetes Reports 201515 4. (https://doi.org/10.1007/s11892-014-0570-5)

13 Balsells M, García-Patterson A, Solà I, Roqué M, Gich I \& Corcoy R. Glibenclamide, metformin, and insulin for the treatment of gestational diabetes: a systematic review and meta-analysis. BMJ 2015 21 h102. (https://doi.org/10.1136/bmj.h102)

14 Rowan JA, Hague WM, Gao W, Battin MR \& Moore MP, MiG trial investigators metformin versus insulin for the treatment of gestational diabetes.. New England Journal of Medicine 2008358 2003-2015. (https://doi.org/10.1056/NEJMoa0707193)

15 Ijäs H, Vääräsmäki M, Saarela T, Keravuo R \& Raudaskoski T. A follow-up of a randomised study of metformin and insulin in gestational diabetes mellitus: growth and development of the children at the age of 18 months. BJOG: An International Journal of Obstetrics and Gynaecology 2015122 994-1000. (https://doi. org/10.1111/1471-0528.12964)

16 International Association of Diabetes and Pregnancy Study Groups Consensus Panel, Metzger BE, Gabbe SG, Persson B, Buchanan TA, Catalano PA, Damm P, Dyer AR, Leiva Ad, Hod M et al. International association of diabetes and pregnancy study groups recommendations on the diagnosis and classification of hyperglycemia in pregnancy. Diabetes Care 201033 676-682. (https://doi.org/10.2337/dc09-1848)

17 Cole TJ, Williams AF, Wright CM \& RCPCH Growth Chart Expert group Revised birth centiles for weight, length and head circumference in the UK-WHO growth charts.. Annals of
Human Biology 201138 7-11. (https://doi.org/10.3109/03014460. 2011.544139)

18 World Health Organization (WHO) and International Diabetes Federation (IDF) Technical Advisory Group. Definition and diagnosis of diabetes mellitus and intermediate hyperglycemia, 2006. (available from: http://www.who.int/diabetes/publications/en/)

19 Moore LE, Clokey D, Rappaport VJ \& Curet LB. Metformin compared with glyburide in gestational diabetes: a randomized controlled trial. Obstetrics and Gynecology 2010115 55-59. (https://doi.org/10.1097/ AOG.0b013e3181c52132)

20 Tertti K, Ekblad U, Vahlberg T \& Rönnemaa T. Comparison of metformin and insulin in the treatment of gestational diabetes: a retrospective, case-control study. Review of Diabetic Studies $2008 \mathbf{5}$ 95-101. (https://doi.org/10.1900/RDS.2008.5.95)

21 Niromanesh S, Alavi A, Sharbaf FR, Amjadi N, Moosavi S \& Akbari S. Metformin compared with insulin in the management of gestational diabetes mellitus: a randomized clinical trial. Diabetes Research and Clinical Practice. 201298 422-429. (https://doi.org/10.1016/j. diabres.2012.09.031)

22 Mcgrath RT, Glastras SJ, Hocking S \& Fulcher GR. Use of metformin earlier in pregnancy predicts supplemental insulin therapy in women with gestational diabetes. Diabetes Research and Clinical Practice. 2016 116 96-99. (https://doi.org/10.1016/j.diabres.2016.04.051)

23 Spaulonci CP, Bernardes LS, Trindade TC, Zugaib M \& Francisco RPV. Randomized trial of metformin vs insulin in the management of gestational diabetes. American Journal of Obstetrics and Gynecology 2013209 34.e1-34.e7. (https://doi.org/10.1016/j.ajog.2013.03.022)

24 Hughes RCE, Moore MP, Gullam JE, Mohamed K \& Rowan J. An early pregnancy $\mathrm{HbA}_{1 \mathrm{c}} \geq 5.9 \%(41 \mathrm{mmol} / \mathrm{mol})$ is optimal for detecting diabetes and identifies women at increased risk of adverse pregnancy outcomes. Diabetes Care 201437 2953-2959 (https://doi. org/10.2337/dc14-1312)

25 Ainuddin J, Karim N, Hasan AA \& Naqvi SA. Metformin versus insulin treatment in gestational diabetes in pregnancy in a developing country. A randomized control trial. Diabetes Research and Clinical Practice 2015107 290-299. (https://doi.org/10.1016/j. diabres.2014.10.001)

26 Tertti K, Ekblad U, Koskinen P, Vahlberg T \& Rönnemaa T. Metformin vs. insulin in gestational diabetes. A randomized study characterizing metformin patients needing additional insulin. Diabetes, Obesity and Metabolism 201315 246-251. (https://doi.org/10.1016/j. diabres.2014.10.001)

27 Pellonperä O, Rönnemaa T, Ekblad U, Vahlberg T \& Tertti K. The effects of metformin treatment of gestational diabetes on maternal weight and glucose tolerance postpartum - a prospective follow-up study. Acta Obstetricia et Gynecologica Scandinavica 201695 79-87.

Received 17July2017

Revised version received 170ctober2017

Accepted 250ctober2017 